\title{
Forecast Share Price Using Technical analysis Tool
}

\author{
*Dr.S.Saravanan ${ }^{1}, * \mathrm{U}$ D Gokul ${ }^{2}$ \\ Department of management studies, Anna university, Trichy, Tamilnadu, India \\ ${ }^{1}$ saravanan@aubit.edu.in, ${ }^{2}$ udgokul1402@gmail.com
}

\begin{abstract}
In recent years many of us have been interesting in investing in the share market. This study focuses on the prediction of share price using a technical analysis tool (candlestick chart). In this study we use 30 companies' one-year data of share prices, top companies of the individual sector in NSE (National Stock Exchange) to forecast the share prices. The sample charts are taken from moneycontrol.com. In this study, we predict the share price using the previous price changes in the share market. Compare them with an actual price, find how many decisions are correct, how many decisions are wrong.
\end{abstract}

Keywords: Technical analysis tool, Candlestick charts, Data prediction, Forecast share price.

\section{Introduction}

This study refers to technical analysis. Technical analysis is a tool used for the prediction of the future share price. This study explains, that how past and present price action in a market and help to determine its future direction. There are a lot of tools under the technical analysis. In this study, we used a candlestick chart to predict the share prices. Candlestick chart is a chart it describes the share price moving clearly. The goal behind the technical analysis is usually to identify trading opportunities and capitalize on them. The objective of the study is to collect data on share prices, formulate candlestick charts, and forecast share prices. In this study we use 30 companies' one-year data of share prices, top companies of the individual sector in NSE (National Stock Exchange) to forecast the share prices. NSE is one of the big stock exchange platform in India.

\section{Review of Literature}

Saravanan S \& Vidhyadharan R (2020) a study on performance evaluation of NSE financial expert around the world have developed numerous approaches to estimate the expected return of stock. Speculation made on securities dominatingly expanding with developing enthusiasm on savings and their requirement for an extra income. Financial specialist are prepared to invest on securities to create exceptional yield ${ }^{[1]}$, yet the vulnerability in the financial exchange is being an obstacle for them to do as such. Zurich (2019) defined Technical analysis "Technical analysis is the study of financial market action. The technician looks at price changes that occur on a day-to-day or week-to-week basis or over any other constant time period displayed in graphic form, called charts. Hence the name chart analysis ${ }^{[2]}$." Plindia (2013) Defined Technical analysis "Technical analysis is a method of forecasting the future movement of share prices by studying past market activity like prices and volumes, using charts and other financial tools. Technical analysis follows the philosophy that past market activity indicates future market activity ${ }^{[3]}$. There are several benefits of using technical analysis." Anne Sraders (2019) Defined Technical analysis "Technical analysis is the process of examining a stock or security's price movements, trading volume and trends to determine how or when to trade it and predict its price movements. Murphy \& John (1999) Defined Technical analysis "Technical analysis is the study of market action, primarily through the use of charts, for the purpose of forecasting future price trends" [4].

\section{Research Methodology}

\section{Sample Design}

Sample Size

30 companies 1 year data of share prices,

Top companies of individual sector in NSE (National Stock Exchange)

Source: Samples are collected from Moneycontrol.com website.

\section{Data analysis and Interpretation}




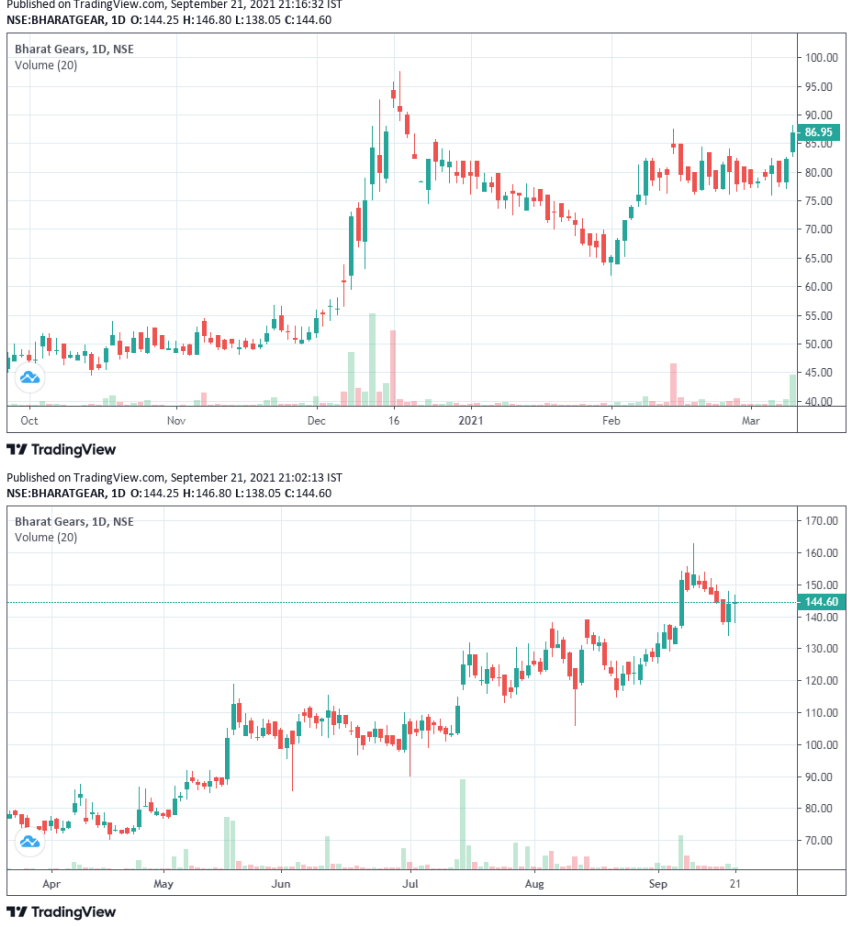

Figure 2. Bharat Gears April 2021 - September 2021

The above candlestick charts of Bharat Gears (October 2020April2021) Trend lines are as

Follows:

October to December 4 - Flat trend

December 5 to December 14 - Up trend

December 15 to February 1 - Down trend

February 2 to September - Uptrend

The above candlestick charts ends with Bullish Star. It means opening and closing prices of the share must be same. September 21 share price starts with Rs.144.25 \& ends with Rs.144.60.

So the next day share price will go down. It showed that it is time to buy shares at a lower price. The buy order may be given at Rs.142.

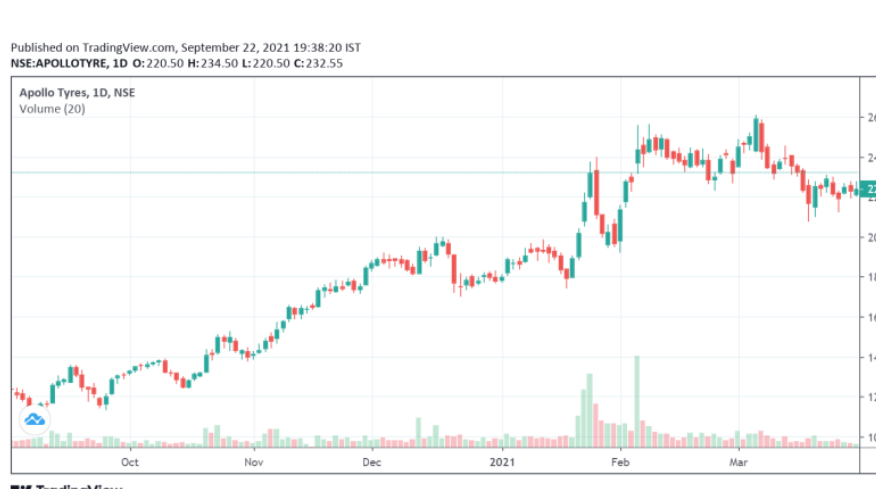

Figure 3. Apollo Tyres October 2020 - March 2021

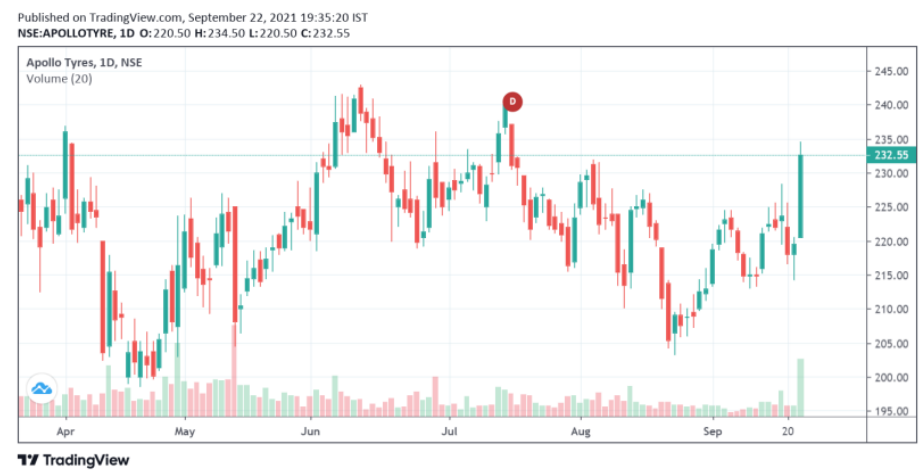

Figure 4. Apollo Tyres April 2021 - September 2021

The above candlestick charts of Apollo Tyres (October 2020April 2021) Trend lines are as follow:

October to March - Up trend

After March, Trend reversal was made \&amp; Goes ups and downs.

The above candlestick chart ends with Huge Bullish candlestick on September 22 (Starts with

Rs.220.50 \& ends with Rs.232.55).

So the next day share price will confirm arising \&amp; Formed a "Three white Soldiers". It showed that it is to sell shares at high profit. The selling order may be given at Rs.237.

\section{Findings}

Following table data's are predicted as same as what we predicted for the above charts. The same method was following.

Table 1. Comparison table for Predicted share price $\&$ Market share price.

$\begin{array}{lllll}\text { S.no } & \text { Company } & \text { Predict } & \text { Market } & \text { Decisi } \\ \text { name } & \text { ed } & \text { share } & \text { on } \\ & & \text { phare } & \text { price } & \\ & & & \\ & & & & \\ & & \text { Decreas } & \text { Decrease } & \\ \text { 1. } & \text { Bharat } & \text { e } & & \\ & \text { Gears } & & \text { Op. } & \text { Correc } \\ & & & \text { Rs.146.15 } & \mathrm{t}\end{array}$




\begin{tabular}{|c|c|c|c|c|c|c|c|c|c|}
\hline & & Op. & $\&$ & & & & & Op. & Correc \\
\hline & & Rs.146 & Cl.Rs.144. & & & & Op. & Rs.90.70 & $\mathrm{t}$ \\
\hline & & $\&$ & 40 & & & & Rs.91 & $\&$ & \\
\hline & & Cl. & & & & & $\&$ & $\mathrm{Cl}$. & \\
\hline & & Rs.142 & & & & & $\mathrm{Cl}$. & Rs.91.35 & \\
\hline & & & & & & & Rs.93 & & \\
\hline & & Increas & Increase & & & & Increas & Decrease & \\
\hline \multirow[t]{8}{*}{2.} & Apollo & e & & & & & e & & \\
\hline & tyres & & Op. & Correc & 7. & Neogen & & Op. & Wrong \\
\hline & & Op. & Rs. 234.65 & $\mathrm{t}$ & & & Op. & Rs. 1327 \& & \\
\hline & & Rs.235 & $\&$ & & & & Rs.132 & $\mathrm{Cl}$. & \\
\hline & & $\&$ & $\mathrm{Cl}$. & & & & $8 \&$ & Rs. 1226.10 & \\
\hline & & $\mathrm{Cl}$. & Rs.236.95 & & & & $\mathrm{Cl}$. & & \\
\hline & & Rs.237 & & & & & Rs.133 & & \\
\hline & & Increas & Increase & & & & 2 & & \\
\hline \multirow[t]{8}{*}{3.} & LIC & $\mathrm{e}$ & & & & & & & \\
\hline & Housing & & Op. & Correc & & & Increas & Decrease & \\
\hline & Finance & Op. & Rs. 435.30 & $\mathrm{t}$ & & & $\mathrm{e}$ & & \\
\hline & & Rs.435 & $\&$ & & 8. & Linde & & Op. & Wrong \\
\hline & & $\&$ & $\mathrm{Cl}$. & & & India & Op. & Rs. $2856 \&$ & \\
\hline & & Cl.Rs.4 & Rs.440.85 & & & & Rs.285 & Cl. & \\
\hline & & 40 & & & & & $0 \&$ & Rs.2799.95 & \\
\hline & & & & & & & $\mathrm{Cl}$. & & \\
\hline \multirow[t]{4}{*}{4.} & Manappur & Rejecte & Rejected & Reject & & & Rs.290 & & \\
\hline & am Fin & d & & ed & & & 0 & & \\
\hline & & Increas & & & & & Increas & Increase & \\
\hline & & $\mathrm{e}$ & --- & & 9. & Crown & $\mathrm{e}$ & & Correc \\
\hline \multirow[t]{7}{*}{5.} & CCCL & & & No & & Lifters & & Op. & $\mathrm{t}$ \\
\hline & & Op. & & Result & & & Op. & Rs. 116.20 & \\
\hline & & Rs.0.50 & & & & & Rs. 115 & $\&$ & \\
\hline & & $\&$ & & & & & $\&$ & Cl. & \\
\hline & & $\mathrm{Cl}$, & & & & & $\mathrm{Cl}$. & Rs.116.95 & \\
\hline & & Rs.0.62 & & & & & Rs.117 & & \\
\hline & & Increas & Increase & & & & & & \\
\hline 6. & Man Infra & e & & & & & & & \\
\hline
\end{tabular}


Forecast Share Price Using Technical analysis Tool

\begin{tabular}{|c|c|c|c|c|c|c|c|c|c|}
\hline \multirow[t]{4}{*}{10.} & NESCO & Rejecte & Rejected & Reject & 14. & Marico & Increas & & \\
\hline & & $\mathrm{d}$ & & ed & & & $\mathrm{e}$ & Op. Rs.570 & \\
\hline & & & & & & & & $\&$ & \\
\hline & & & & & & & Op. & $\mathrm{Cl}$. & \\
\hline \multirow[t]{10}{*}{11.} & Butterfly & Increas & Increase & Correc & & & Rs.570 & Rs. 566.80 & \\
\hline & & $\mathrm{e}$ & & $\mathrm{t}$ & & & $\&$ & & \\
\hline & & & Op. & & & & $\mathrm{Cl}$. & & \\
\hline & & Op, & Rs.934.90 & & & & Rs.578 & & \\
\hline & & Rs.933 & $\&$ & & & & Increas & Decrease & \\
\hline & & $\&$ & $\mathrm{Cl}$. & & 15. & GMR Infra & $\mathrm{e}$ & & Wrong \\
\hline & & $\mathrm{Cl}$. & Rs.971.25 & & & & & Op. & \\
\hline & & Rs.968 & & & & & Op. & Rs.36.40 & \\
\hline & & & & & & & Rs.38 & $\&$ & \\
\hline & & & & & & & $\&$ & $\mathrm{Cl}$. & \\
\hline \multirow[t]{11}{*}{12.} & Bajaj & Decreas & Increase & Wrong & & & $\mathrm{Cl}$. & Rs.36.00 & \\
\hline & Electric & e & & & & & Rs.41.7 & & \\
\hline & & & Op. & & & & 5 & & \\
\hline & & Op. & Rs.1519.95 & & & & Decreas & Decrease & \\
\hline & & Rs.151 & $\&$ & & 16. & BHEL & $\mathrm{e}$ & & Correc \\
\hline & & $8 \&$ & Cl.Rs.1531 & & & & & Op. & $\mathrm{t}$ \\
\hline & & $\mathrm{Cl}$. & .80 & & & & Op. & Rs. 57.50 & \\
\hline & & Rs.151 & & & & & Rs. 58 & $\&$ & \\
\hline & & 0 & & & & & $\&$ & $\mathrm{Cl}$. & \\
\hline & & & & & & & $\mathrm{Cl}$. & Rs.57.15 & \\
\hline & & & & & & & Rs.57.5 & & \\
\hline \multirow[t]{10}{*}{13.} & Bhartiya & Decreas & Increase & Wrong & & & Increas & Increase & \\
\hline & Inter & $\mathrm{e}$ & & & 17. & Pioneer & $\mathrm{e}$ & & Correc \\
\hline & & & Op. Rs. 218 & & & Distill & & Op. & $\mathrm{t}$ \\
\hline & & Op. & $\&$ & & & & Op. & Rs. 157.70 & \\
\hline & & Rs. 215 & $\mathrm{Cl}$. & & & & Rs. 158 & $\&$ & \\
\hline & & $\&$ & Rs. 229.10 & & & & $\&$ & $\mathrm{Cl}$. & \\
\hline & & $\mathrm{Cl}$. & & & & & $\mathrm{Cl}$. & Rs.169.95 & \\
\hline & & Rs.198 & & & & & Rs.173 & & \\
\hline & & & & & & & Increas & Increase & \\
\hline & & & Decrease & Wrong & 18. & & $\mathrm{e}$ & & \\
\hline
\end{tabular}




\begin{tabular}{|c|c|c|c|c|}
\hline & Andhra & & Op. & Correc \\
\hline & Sugar & Op. & Rs.577.30 & $\mathrm{t}$ \\
\hline & & Rs.576 & $\&$ & \\
\hline & & $\&$ & $\mathrm{Cl}$. & \\
\hline & & $\mathrm{Cl}$. & Rs.574.35 & \\
\hline & & Rs.582 & & \\
\hline \multirow[t]{3}{*}{19.} & Kotak MF- & Rejecte & Rejected & Reject \\
\hline & GETF & d & & ed \\
\hline & & Decreas & Decrease & \\
\hline \multirow[t]{9}{*}{20.} & SBI Gold- & $\mathrm{e}$ & & Correc \\
\hline & GETF & & Op. & $\mathrm{t}$ \\
\hline & & Op. & Rs.4169.95 & \\
\hline & & Rs.417 & $\&$ & \\
\hline & & 0 & $\mathrm{Cl}$. & \\
\hline & & $\&$ & Rs. 4136.25 & \\
\hline & & $\mathrm{Cl}$. & & \\
\hline & & Rs.415 & & \\
\hline & & 0 & & \\
\hline
\end{tabular}

\begin{tabular}{lllll}
\hline 2 & Op. Rs. & Op. & \\
2 & $16 \&$ & Rs.16.05 & \\
$\cdot$ & & Cl. Rs. & $\&$ & \\
& & 14.5 & Cl. & \\
& & & Rs.15.45 & \\
& & Increas & Decrease & \\
. Soril Infra & e & & Wrong \\
& Ind & & Op. &
\end{tabular}




\begin{tabular}{|c|c|c|c|c|}
\hline & & Op. & Op. & \\
\hline & & Rs.364 & Rs.362.50 & \\
\hline & & $\&$ & $\&$ & \\
\hline & & Cl. & $\mathrm{Cl}$. & \\
\hline & & Rs.345 & Rs.348.55 & \\
\hline & & Increas & Decrease & \\
\hline \multirow[t]{8}{*}{27.} & Usha & $\mathrm{e}$ & & Wrong \\
\hline & Martin & & Op. & \\
\hline & & Op. & Rs. 80.10 & \\
\hline & & Rs. 82 & $\&$ & \\
\hline & & $\&$ & $\mathrm{Cl}$. & \\
\hline & & Cl. & Rs.77.60 & \\
\hline & & Rs.86 & & \\
\hline & & Increas & Increase & \\
\hline \multirow[t]{7}{*}{28.} & Jai Corp & $\mathrm{e}$ & Op. & Correc \\
\hline & & Op. & Rs. 132.10 & $\mathrm{t}$ \\
\hline & & Rs.133 & $\&$ & \\
\hline & & $\&$ & $\mathrm{Cl}$. & \\
\hline & & $\mathrm{Cl}$. & Rs. 135.40 & \\
\hline & & Rs.137 & & \\
\hline & & Decreas & Increase & \\
\hline \multirow[t]{6}{*}{29.} & ONGC & $\mathrm{e}$ & Op. & Wrong \\
\hline & & Op. Rs. & Rs. 134.80 & \\
\hline & & $135 \&$ & $\&$ & \\
\hline & & $\mathrm{Cl}$. & $\mathrm{Cl}$. & \\
\hline & & Rs.133 & Rs. 137.75 & \\
\hline & & Increas & Decrease & \\
\hline \multirow[t]{7}{*}{30.} & Oil India & $\mathrm{e}$ & & Wrong \\
\hline & & & Op. Rs.221 & \\
\hline & & Op. & $\&$ & \\
\hline & & Rs.221 & $\mathrm{Cl}$. & \\
\hline & & $\&$ & Rs.213.85 & \\
\hline & & Cl. Rs. & & \\
\hline & & 226 & & \\
\hline
\end{tabular}

\section{Conclusion and Suggestion}

In this study, sample sizes of 30 companies with 1 year of data on share prices were considered for analysis. The prediction status is out of thirty companies, 15 companies share prices are correctly predicted, 11 companies share prices are wrongly predicted, 3 companies will be rejected and 1 company status is unsolved. So the correct prediction possibility is worked out as 0.5 . This will lead to the conclusion that prediction using candle stick charts were reliable and the accuracy can be increased with increase in sample size.

\section{References}

[1]. Saravanan $\mathrm{S}$ and Vidhyatharan R., "INTERPRETATION OF STOCK BETA IN NATIONAL STOCK EXCHANGE" Pramana Research Journal Vol-10, Issue-2, 2020. Pp123-133.

[2]. Zurich "Global Technical Research and Behavioral Finance",e-book,2021 p.3.

[3]. Plindia "MACD Indicator", e-copy published by Knowledge center e-mailer, 2013, P.1.

[4]. Anne sraders, "What is technical analysis? Definition, basics and Example e-journal, 2019.

[5]. Murphy \& John, "Technical analysis of the Financial markets”, e-book, 1999. 